\title{
Symmetries and fixed point stability of stochastic differential equations modeling self-organized criticality
}

\author{
Álvaro Corral* and Albert Díaz-Guilera ${ }^{\dagger}$ \\ Departament de Física Fonamental, Facultat de Física, Universitat de Barcelona, Diagonal 647, E-08028 Barcelona, Spain
}

(Received 15 October 1996)

\begin{abstract}
A stochastic nonlinear partial differential equation is constructed for two different models exhibiting selforganized criticality: the Bak-Tang-Wiesenfeld (BTW) sandpile model [Phys. Rev. Lett. 59, 381 (1987); Phys. Rev. A 38, 364 (1988)] and the Zhang model [Phys. Rev. Lett. 63, 470 (1989)]. The dynamic renormalization group (DRG) enables one to compute the critical exponents. However, the nontrivial stable fixed point of the DRG transformation is unreachable for the original parameters of the models. We introduce an alternative regularization of the step function involved in the threshold condition, which breaks the symmetry of the BTW model. Although the symmetry properties of the two models are different, it is shown that they both belong to the same universality class. In this case the DRG procedure leads to a symmetric behavior for both models, restoring the broken symmetry, and makes accessible the nontrivial fixed point. This technique could also be applied to other problems with threshold dynamics. [S1063-651X(97)04603-5]
\end{abstract}

PACS number(s): 64.60.Ak, 05.40.+j, 05.90.+m, 64.60.Lx

\section{INTRODUCTION}

In the past decade much attention has been paid to the phenomenon known as self-organized criticality (SOC). Bak, Tang, and Wiesenfeld [1] studied a cellular automaton model as a paradigm for the explanation of two widely occurring phenomena in nature: $1 / f$ noise and fractal structures. Both have in common a lack of characteristic scales. Although the SOC models do not always show $1 / f$ noise, they have no characteristic scales either; this scale invariance suggests that these systems are critical in analogy with classical equilibrium critical phenomena; but in SOC one deals with dynamical nonequilibrium statistical properties. Moreover, the system evolves naturally to the critical state without any tuning of external parameters, that is, in a self-organized process.

Several cellular automata and coupled map lattices models exhibiting SOC have been reported in the literature. In the original sandpile model of Bak et al. [1], the system is perturbed externally by a random addition of sand grains. Once the slope between neighboring cells has reached a threshold value, sand is transferred between them in a fixed amount. Taking this model as a reference, different dynamical rules have been investigated, leading to a wide variety of universality classes. Continuous variables with a full transfer from a cell instead of a fixed discrete amount [2-5]; directed flows [6]; a threshold condition imposed on the height, on the gradient, or even on the Laplacian [7]; and anisotropy [8] are a few examples. These randomly driven models do not exhibit SOC when the interaction rules are not conservative [9]. Later on, other deterministically driven models have been introduced where conservation is not a necessary condition [10-15]. Much more recently, sandpile models with deterministic perturbations but intrinsic randomness in the threshold dynamics have been used to reproduce experimen-

\footnotetext{
*Electronic address: alvaro@ulyses.ffn.ub.es

†Electronic address: albert@ulyses.ffn.ub.es
}

tal results on transport properties on rice piles [16]. The close connection between these sandpile models and interface depinning has been established in Ref. [17].

Some authors have attempted to connect the randomly driven models to stochastic differential equations $[18,19]$. These continuous descriptions are developed according to the symmetry rules obeyed by the discrete models in order to achieve a generic scale-invariant condition [20]. Nevertheless, none of them either explicitly or implicitly includes the threshold condition, which is one of the main characteristics of SOC models. On the other hand, anomalous diffusion equations with singularities in the diffusion coefficient have been considered in order to study the deterministic dynamics of the avalanches generated in the critical state [21,22]. A different approach has been introduced by Pietronero and co-workers, using a real-space renormalization procedure to determine the dynamical exponent as well as the avalanche size exponent [23].

In a previous paper [24] one of us studied two nonlinear stochastic differential equations derived from the discrete dynamical rules of two models with different symmetry properties. In principle, one would expect, for this reason, different critical behavior. However, it was shown analytically, by means of the dynamic renormalization group (DRG) [2527], that both models belong to the same universality class. The threshold condition was kept, but the step function was regularized in order to allow a power-series expansion. In the limit that recovers the threshold it was shown that the coupling constants that distinguish both models become decoupled from the common coupling constants; since the critical exponents depend only on the latter constants, one obtains the same values for both models. Once this equivalence was established, the most symmetric model was considered, showing that an infinite number of coupling constants was relevant below the upper critical dimension $d_{c}=4$; by expanding the number of nonlinearities, the DRG procedure, up to first order in $\epsilon=4-d$, gave an estimate of the dynamical exponent close to the value obtained by scaling arguments and in the numerical simulations $[2,5]$. This value of 
the dynamical exponent is obtained when the flow in parameter space reaches the nontrivial stable fixed point; nevertheless, when taking into account the physical values of the parameters, they do not lie in the basin of attraction of the fixed point, thus making this computed value in some sense speculative since it cannot be ensured that the flow in parameter space will be able to reach the attractor.

Our goal in this paper is to complement the previous work in order to check the validity of the calculation of the critical exponents at the stable fixed points and to analyze the role played by symmetries in randomly driven SOC models and, in general, in other models where an infinite hierarchy of nonlinear terms is required. Our procedure also illustrates the effect of symmetry breaking in DRG calculations as a mechanism to make the attractors in parameter space accessible for the physical values of the parameters in the original equations. The continuum equation for the Bak-TangWiesenfeld (BTW) and Zhang models is introduced in Sec. II, as well as the alternative regularization that breaks the symmetry that distinguish both models. In Sec. III we develop the DRG procedure and show that this symmetry is irrelevant, in view of the fact that the nontrivial fixed point is not modified by this alternative approach. Moreover, the effect of symmetry breaking allows the flow of the original parameters to reach the nontrivial fixed point, where the critical exponents can now be computed. Finally, we present our conclusions in Sec. IV.

\section{MODELS AND SYMMETRIES}

First, we describe briefly the dynamics of the two SOC models under consideration. The first model was originally proposed by Zhang [2] and consists of a $d$-dimensional lattice in which any site can store some continuously distributed variable $E$. This variable, which we will call energy, can have different physical interpretations [3]. The system is perturbed by adding a random amount of energy $\delta E>0$ at a randomly chosen site. Once a site reaches a value of the energy greater than the threshold value $E_{c}$, this site becomes active and transfers all its energy to its nearest neighbors. At this point the input of energy from the outside is turned off. The energy transferred to the neighboring sites can make them active, giving rise to an activation cluster or avalanche, which ends when all the sites have reached a value of the energy smaller than $E_{c}$. It is only when the avalanche has stopped that energy is added again, otherwise the system remains quiescent. In this way there is a clear time-scale separation in the dynamics. The external noise acts in a slow time scale, whereas the avalanches evolve infinitely fast, in comparison. The second model differs from Zhang's model only in the amount of energy an active site transfers to its neighbors, which is a fixed amount $E_{c}$, instead of its whole energy $E$. Therefore it is closer to the original sandpile model of Bak et al. [1], but is continuous in $E$. When $\delta E$ is not random but fixed this difference becomes irrelevant. For this reason, it will be referred to as BTW model. Notice that both models are conservative in the sense that the added energy (always positive) is only dissipated at the open boundaries.

The microscopic evolution rules can be written from time $t$ (on a fast time scale) to $t+1$ for each site $i$ as

$$
\begin{aligned}
E_{i}(t+1)= & E_{i}(t)-E_{i}(t) \Theta\left(E_{i}(t)-E_{c}\right) \\
& +\frac{1}{q} \sum_{\mathrm{NN}} E_{\mathrm{NN}}(t) \Theta\left(E_{\mathrm{NN}}(t)-E_{c}\right)+\xi_{i}(t)
\end{aligned}
$$

and

$$
\begin{aligned}
E_{i}(t+1)= & E_{i}(t)-E_{c} \Theta\left(E_{i}(t)-E_{c}\right) \\
& +\frac{1}{q} \sum_{\mathrm{NN}} E_{c} \Theta\left(E_{\mathrm{NN}}(t)-E_{c}\right)+\xi_{i}(t)
\end{aligned}
$$

for Zhang's and the BTW model, respectively. The sum runs over the $q$ nearest neighbors of site $i$, labeled $\mathrm{NN}$, and the threshold condition enters through the Heaviside step function $\Theta$, defined as $\Theta(x<0)=0$ and $\Theta(x>0)=1$. Due to the continuous nature of the models the value $\Theta(x=0)$ is irrelevant and we can keep it undefined, for now. For the external noise $\xi_{i}(t)$, which drives the system only when there are no active sites, one can formally write

$$
\xi_{i}(t)=\delta E \delta_{i, n(t)} \prod_{\forall j}\left[1-\Theta\left(E_{j}(t)-E_{c}\right)\right]
$$

where $\delta_{i, n(t)}$ is the Kronecker delta symbol and $n(t)$ is a random vector pointing towards the site of the lattice that will be perturbed with a random amount of energy $\delta E$ (in the original BTW model $\delta E=E_{c} / q$ ). The product runs over all the lattice sites.

When applying the DRG one deals with infinite systems and then the important effect of dissipation at the open boundaries is not taken into account. However, in SOC models a distribution of absorbing defects through the lattice plays the same role as the open (absorbing) boundaries [28], as we have verified through computer simulations [29]. Then, we can redefine our models in an infinite lattice, but with a quenched distribution of defects. The results are not modified with this assumption. Another possibility is to consider that each site of an infinite lattice has a small probability of dissipating an amount of energy $E_{c} / q$ when it topples, instead of transferring it to a certain neighbor. This procedure, which represents the assumption of random boundaries, accuracy implies that when a site receives a toppling from some neighbor, it has a small probability of not accepting the amount of energy $E_{c} / q$, which is lost [30]. This dissipation can be included as a new term in the noise, and Eq. (2) has to be replaced by

$$
\begin{aligned}
\xi_{i}(t)= & \delta E \delta_{i, n(t)} \prod_{\forall j}\left[1-\Theta\left(E_{j}(t)-E_{c}\right)\right] \\
& -\sum_{\mathrm{NN}} \zeta_{\mathrm{NN}} \Theta\left(E_{\mathrm{NN}}(t)-E_{c}\right),
\end{aligned}
$$

where $\zeta_{\mathrm{NN}}$ is a dichotomous noise, taking the value 0 with a large probability and the value $E_{c} / q$ (dissipation) with a small one. If $\zeta_{\mathrm{NN}}$ depends on $t$, i.e., $\zeta_{\mathrm{NN}}=\zeta_{\mathrm{NN}}(t)$, we are dealing with annealed random boundaries, whereas if it depends only on the position, we have quenched random boundaries or absorbing defects. 
In terms of a rescaled energy $E-E_{c} \rightarrow E$ and introducing a parameter $Z$ to unify the description, we have for both models

$$
\begin{aligned}
E_{i}(t+1)-E_{i}(t)= & \frac{1}{q} \sum_{\mathrm{NN}}\left\{\left[Z E_{\mathrm{NN}}(t)+E_{c}\right] \Theta\left(E_{\mathrm{NN}}(t)\right)\right. \\
& \left.-\left[Z E_{i}(t)+E_{c}\right] \Theta\left(E_{i}(t)\right)\right\}+\xi_{i}(t),
\end{aligned}
$$

where $Z=1$ for Zhang's model and $Z=0$ for the BTW model. Equation (4) defines a stochastic coupled map lattice. Moreover, notice that the deterministic BTW equation displays invariance under a parity transformation of the order parameter $E \rightarrow-E$. This is the only symmetry that the BTW model does not share with Zhang's model. The common symmetries are invariance under spatial translations, rotations, and reflections, as well as conservation of the order parameter.

Equation (4) can be coarse grained in order to obtain a continuum equation for the effective $E(\vec{r}, t)$. Then, by using the prescriptions for the temporal derivative and for the Laplace operator

$$
\frac{\partial E(\vec{r}, t)}{\partial t}=\alpha \nabla^{2}\left\{\left[Z E(\vec{r}, t)+E_{c}\right] \Theta(E(\vec{r}, t))\right\}+\eta(\vec{r}, t),
$$

where $\alpha$ is a coefficient that depends on the lattice spacing, the unit time step, and the coordination number $q$. The noise $\eta(\vec{r}, t)$ accounts for the effective external noise as well as for the internal noise that appears due to the elimination of microscopic degrees of freedom.

Up to this point, Eq. (5) truly describes the coarse-grained evolution of the system, but we have not yet characterized the noise $\eta(\vec{r}, t)$, which derives from $\xi_{i}(t)$. The product in Eq. (3) makes $\eta(\vec{r}, t)$ a multiplicative noise that depends on the whole lattice state, and the problem is intractable. We are going to ignore the restrictions imposed by the step functions in Eq. (5), thus breaking the time-scale separation. Then the noise $\eta(\vec{r}, t)$ acts continuously in time and can provoke avalanches to overlap. However, for small enough noise this is very unlikely, and one can still identify avalanches in computer simulations. Moreover, the dynamical exponent does not change with this assumption [5] because the added noise is orders of magnitude smaller than the energy transferred by the avalanche and thus its dynamics is not affected. In this case we still have two time scales, although they overlap. In what follows $\eta(\vec{r}, t)$ will be considered as an additive random process including two effects: the external driving, always positive, and the dissipation at the (random) boundaries, always negative. In the statistical stationary state the random input of energy must equal, on average, the output at the boundaries. Then we assume that

$$
\langle\eta(\vec{r}, t)\rangle=0 .
$$

In fact, this is the same assumption made in all the studies of SOC by means of DRG $[18,19]$ and it is somehow equivalent to the stationary condition used in Ref. [23].

Moreover, we are mainly interested in the spatiotemporal propagation of a perturbation through the system, that is, in measuring the value of the dynamical exponent. For this purpose we have to look at the system on a fast time scale, i.e., the scale of the evolution of the avalanches. In Ref. [5] it was argued that in this case one can understand the noise as a quenched Gaussian process uncorrelated in space, and then its correlation function is given by

$$
\left\langle\eta(\vec{r}, t) \eta\left(\overrightarrow{r^{\prime}}, t^{\prime}\right)\right\rangle=2 \Gamma \delta^{d}\left(\vec{r}-\overrightarrow{r^{\prime}}\right) .
$$

When looking at the system on a slow time scale one cannot use this prescription for the noise, which has to be uncorrelated in time too, i.e., $\left\langle\eta(\vec{r}, t) \eta\left(\overrightarrow{r^{\prime}}, t^{\prime}\right)\right\rangle$ $=2 \Gamma \delta^{d}\left(\vec{r}-\overrightarrow{r^{\prime}}\right) \delta\left(t-t^{\prime}\right)$, and this prescription is mainly related to the interface roughness between avalanches [31].

Equations (5)-(7), together with the fact that the noise is a Gaussian process, completely define our model. However, the presence of the step function in Eq. (5) gives rise to a strong nonlinearity. A perturbative expansion of this equation can be performed if one regularizes the step function as

$$
\Theta(E)=\lim _{\beta \rightarrow \infty} f(\beta E)
$$

and makes a series expansion of $f(\beta E)$ in powers of $E$ $[22,24]$. The function $f(x)$ must be monotonical increasing with $f(-\infty)=0$ and $f(\infty)=1$. Moreover, we choose $f(x)-1 / 2$ as an odd function, so $f(0)=1 / 2$. Several functions of this type have been used in the literature, but that coming from the error function as

$$
f(x)=\frac{1+\operatorname{erf}(x)}{2}=\frac{1}{\sqrt{\pi}} \int_{-\infty}^{x} e^{-y^{2}} d y
$$

allows a power expansion that has an infinite radius of convergence, in contrast with previous choices [22,24]. In any case, the relevant results do not depend on the particular form of $f(x)$.

The regularization given by Eq. (8) keeps the symmetry of the step function and therefore the invariance under a parity transformation in the BTW model. As an alternative regularization that breaks this invariance we propose

$$
\Theta(E)=\lim _{\beta \rightarrow \infty} f(\beta E+K)
$$

with $K$ an arbitrary constant. Although in the limit $\beta \rightarrow \infty$ we recover the step function, we do not recover its symmetry anymore because $\Theta(E=0)=f(K) \neq 1 / 2$ if $K \neq 0$, and this is the reason for the breaking of the symmetry in the BTW model. Now we perform a series expansion of the regularizing function $f(\beta E)$ in powers of $E$, obtaining

$$
\Theta(E)=\lim _{\beta \rightarrow \infty} \sum_{n=0}^{\infty} a_{n}(\beta, K) E^{n}
$$

where the coefficients $a_{n}(\beta, K)$ are given by

$$
a_{n}(\beta, K)=\frac{f^{(n)}(K) \beta^{n}}{n !},
$$


with $f^{(n)}(K)$ being the $n$ th-order derivative of $f(x)$ at $x=K$. Substituting the expansion (11) into Eq. (5), we can write

$\frac{\partial E(\vec{r}, t)}{\partial t}=D \nabla^{2} E(\vec{r}, t)+\sum_{n=2}^{\infty} \lambda_{n} \nabla^{2} E^{n}(\vec{r}, t)+\eta(\vec{r}, t)$,

where the effective diffusion constant $D$ and the coupling constants $\lambda_{n}$ (which make the equation nonlinear) take different values depending on the model:

$$
\begin{array}{r}
D=\lim _{\beta \rightarrow \infty} \alpha\left(E_{c} f^{(1)}(K) \beta+Z f(K)\right), \\
\lambda_{n}=\lim _{\beta \rightarrow \infty} \frac{\alpha \beta^{n}}{n !}\left(E_{c} f^{(n)}(K)+Z \frac{n f^{(n-1)}(K)}{\beta}\right), \\
n=2,3, \ldots, \infty .
\end{array}
$$

On the one hand, for $K=0$, since all the even derivatives verify $f^{(2 n+2)}(0)=0$, all even coupling constants vanish for the BTW model, whereas they do not for the Zhang model. Using Eq. (13), this allows one to verify the symmetry of the BTW model under the parity transformation of the order parameter $E$. On the other hand, for $K \neq 0$, the even coupling constants do not vanish in any case and this constitutes the symmetry breaking for the BTW model. Then, under this condition, the only difference between both models is that the constants depend on $\beta$ in a different way; however, it is easy to see that in the limit $\beta \rightarrow \infty$ both sets of constants are identical and then the Zhang model and the brokensymmetry BTW model have to belong to the same universality class. This can only be shown for $K \neq 0$. Nevertheless, considering $K=0$ only introduces a difference in the value of $\Theta(0)$, which is irrelevant in a continuous model, and then one can include the (symmetric) BTW model in this universality class too.

At this point it is worth noting that we have transformed a stochastic coupled map lattice, which involves a threshold condition and presents a clear separation of time scales, into a nonlinear stochastic partial differential equation, where the nonlinearity of the threshold is described by an infinite series of powers and the randomness enters via a Gaussian process, with zero mean to account for the dissipation at the boundaries. During this transformation, and due to the approximations we have performed concerning the noise correlation, we have broken the time-scale separation since the noise acts constantly in time. Nevertheless, we expect that such an equation explains the dynamical properties of the system within the fast time scale of the propagation of the avalanches. As we have mentioned before and as discussed in [31], to deal with the slow time scale, where the avalanches are instantaneous, another noise correlation is more appropriate.

\section{DYNAMIC RENORMALIZATION-GROUP PROCEDURE}

The model to be studied by the DRG is defined by the nonlinear partial differential equation (13) and the Gaussian noise given by Eqs. (6) and (7). As a first step we can check the relevance of the different coupling constants in this equation by naive dimensional analysis: a change of scale $b=e^{l}>1$,

$$
\overrightarrow{r^{\prime}}=e^{-l} \vec{r}, \quad t^{\prime}=e^{-z l} t, \quad E^{\prime}=e^{-\chi l} E,
$$

is performed in Eqs. (13) and (7), with $\chi$ being the roughness exponent, which is related to the hydrodynamic exponent, and $z$ the dynamical exponent. Then one obtains that the parameters transform as

$$
D \rightarrow b^{z-2} D, \quad \Gamma \rightarrow b^{2(z-\chi)-d} \Gamma, \quad \lambda_{n} \rightarrow b^{z+(n-1) \chi-2} \lambda_{n} .
$$

Under this scaling transformation, $z$ and $\chi$ are chosen to keep the linear model scale invariant, i.e., the parameters $D$ and $\Gamma$ have not to be modified. This choice gives $z=2$, $\chi=(4-d) / 2$, and

$$
\lambda_{n} \rightarrow b^{(4-d)(n-1) / 2} \lambda_{n}
$$

Then one can see that when we apply iteratively the transformation $(b \rightarrow \infty)$ for $d>4$ all the nonlinear terms vanish and are irrelevant. However, all the coupling constants go to infinity for $d<4$ and hence all nonlinear terms become relevant; this implies that the upper critical dimension is $d_{c}=4$ and nontrivial values of the exponents are expected below it.

The relevance of all the terms makes our problem much more complicated than, for instance, the Kardar-ParisiZhang model of interface growth, where only the first nonlinear term is relevant [32]. The appropriate treatment of Eq. (13) would be to renormalize the infinite number of relevant coupling constants that are involved. Of course this is impossible to do in practice. In [24] an expansion in the number of coupling constants for the BTW model was performed with only odd terms, i.e., without symmetry breaking $(K=0)$. The critical exponents where obtained as a function of the highest coupling constant, up to $\lambda_{9}$. Fortunately, the dynamical exponent was well behaved and could be extrapolated up to $\lambda_{\infty}$. However, keeping the symmetry of the step function, the nontrivial fixed point of the DRG is unreachable using the parameters given by Eqs. (14) and (15), even for the Zhang model. We want to show that with the proposed alternative regularization of the step function, which breaks the symmetry of the BTW model and allows the existence of even coupling constants, the DRG fixed points are not changed, but now they are accessible to the flow when the parameters take their real values. For this reason, and as an initial attempt to justify our hypothesis as well as the conclusions of Ref. [24], we will focus on Eq. (13) with only its first two nonlinear terms, i.e., $\lambda_{2}$ and $\lambda_{3}$, and see how they behave under a DRG transformation,

$$
\begin{aligned}
\frac{\partial E(\vec{r}, t)}{\partial t}= & D \nabla^{2} E(\vec{r}, t)+\lambda_{2} \nabla^{2} E^{2}(\vec{r}, t) \\
& +\lambda_{3} \nabla^{2} E^{3}(\vec{r}, t)+\eta(\vec{r}, t) .
\end{aligned}
$$

The DRG procedure consists of the removal of the fast modes (large wave number $k$ ) in the momentum space, followed by a rescaling of a factor $e^{l}$ in order to recover the 

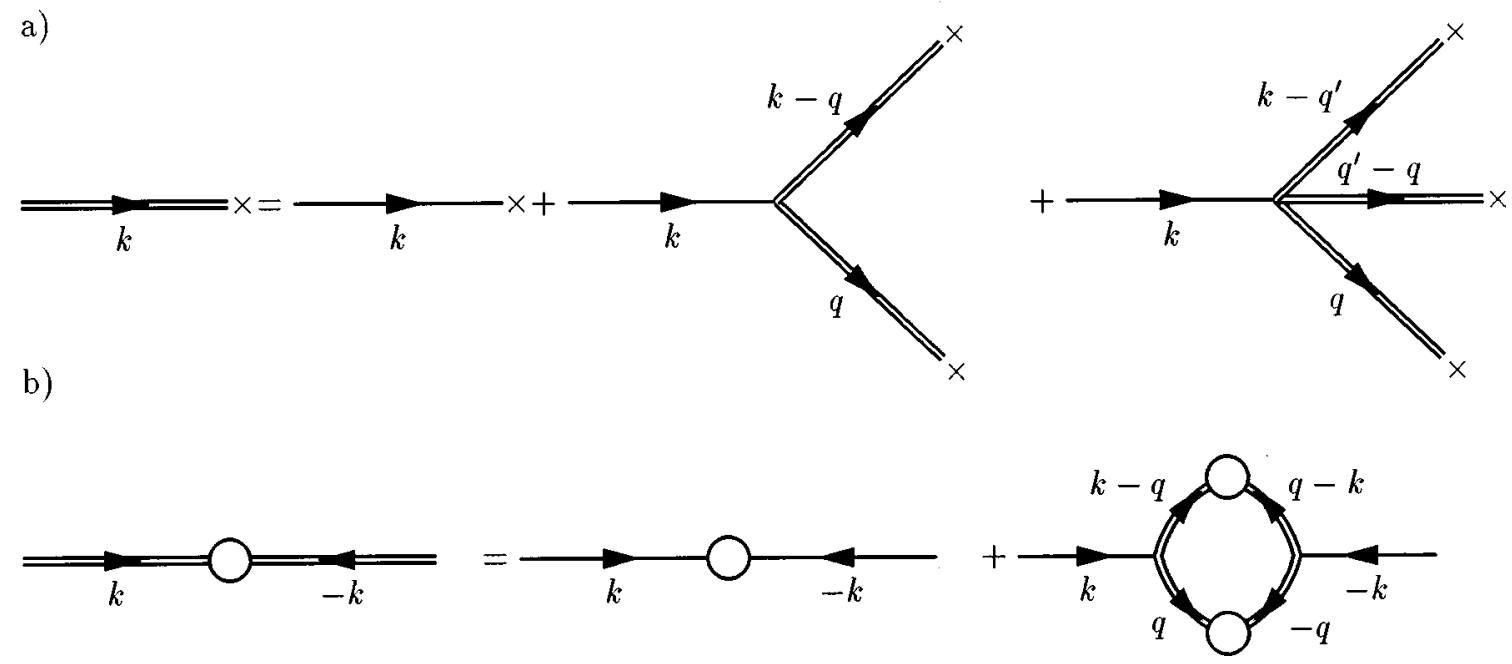

FIG. 1. Diagrammatic expressions for Eqs. (21) and (25), defined in the range $0<k<\Lambda$. The double bar with the cross $\times$ at its end is the order parameter $E$, the single bar with the cross represents $G_{0} \eta$, whereas the single bar alone is $G_{0}$. A vertex with $n$ branches $(n=2$ or 3 in the figure) represents a convolution product of $n$ elements, including a prefactor $-\lambda_{n} k^{2} /(2 \pi)^{(n-1)(d+1)}$. The circles correspond to the average over the noise.

original Brillouin zone [25-27]. After this transformation, one obtains an equation that is equivalent to the original one, but with different (effective or renormalized) coefficients. Successive iterations of this transformation give the flow of the coefficients in the parameter space. If this flow converges towards a fixed point, the system presents "scale invariance" in the hydrodynamic limit (large-distance and longtime behavior). Then, the fluctuations of the order parameter verify the scaling equation

$$
\left\langle\left[E\left(\vec{r}_{0}, t_{0}\right)-E\left(\vec{r}_{0}+\vec{r}, t_{0}+t\right)\right]^{2}\right\rangle^{1 / 2} \sim r^{\chi} F\left(t / r^{z}\right),
$$

where the critical exponents $\chi$ and $z$ are those that ensure the existence of the fixed point. However, it is worth mentioning that with this procedure the scaling function $F(x)$ remains unknown [33].

We now outline the DRG calculation. First of all we write Eq. (19) in Fourier space

$$
E=G_{0} \eta-G_{0} \frac{\lambda_{2} k^{2}}{(2 \pi)^{d+1}} E^{*} E-G_{0} \frac{\lambda_{3} k^{2}}{(2 \pi)^{2(d+1)}} E^{*} E^{*} E .
$$

Here $E(\vec{k}, \omega)$ and $\eta(\vec{k}, \omega)$ are defined as the Fourier transforms of $E(\vec{r}, t)$ and $\eta(\vec{r}, t)$, i.e.,

$$
E(\vec{k}, \omega)=\int d^{d} r d t e^{i(\omega t-\vec{k} \cdot \vec{r})} E(\vec{r}, t),
$$

whereas

$$
G_{0}(k, \omega)=\frac{1}{-i \omega+D k^{2}}
$$

is called the bare propagator. The symbol $*$ represents the convolution product, defined as

$$
(E * E)(\vec{k}, \omega)=\int d^{d} q d \Omega E(\vec{q}, \Omega) E(\vec{k}-\vec{q}, \omega-\Omega) .
$$

Figure 1(a) shows the expression of Eq. (21) in terms of Feynman diagrams. As the intensity of the noise $\Gamma$ has also to be renormalized by the DRG transformation, we need to consider the equation for the correlation function of the transformed energy $\left\langle E(\vec{k}, \omega) E\left(\overrightarrow{k^{\prime}}, \omega^{\prime}\right)\right\rangle$, which, up to oneloop order, is

$$
\left\langle E E^{\prime}\right\rangle=G_{0} G_{0}^{\prime}\left\langle\eta \eta^{\prime}\right\rangle+\frac{\lambda_{2}^{2} k^{2} k^{\prime 2} G_{0} G_{0}^{\prime}}{(2 \pi)^{2(d+1)}}\left\langle(E * E)(E * E)^{\prime}\right\rangle,
$$

where the prime denotes a dependence on $\vec{k}^{\prime}, \omega^{\prime}$ instead of the dependence on $\vec{k}, \omega$. The diagrammatic representation of this equation is shown in Fig. 1(b). Equations (21) and (25), which are the ones that we are going to renormalize, hold for $0<k<\Lambda$, where $\Lambda$ is the wave-number cutoff due to the underlying discrete structure. The transformed noise $\eta(\vec{k}, \omega)$ turns out to be also a Gaussian process with zero mean, but with a correlation

$$
\left\langle\eta(\vec{k}, \omega) \eta\left(\vec{k}^{\prime}, \omega^{\prime}\right)\right\rangle=2(2 \pi)^{d+2} \Gamma \delta^{d}\left(\vec{k}+\vec{k}^{\prime}\right) \delta(\omega) \delta\left(\omega^{\prime}\right) .
$$

The first step of the DRG transformation consists in splitting the Fourier space in two shells: an inner shell, which contains the slow modes, i.e., $0<k<e^{-l} \Lambda$, and an outer shell, containing the fast modes, $e^{-l} \Lambda<k<\Lambda$. Both modes are coupled through the convolution products in Eqs. (21) and (25). We consider the diagrams for the slow modes and perform a perturbative expansion of the fast ones up to the lowest order in the intensity of the noise (see the Appendix for more details). Then we integrate out these modes by an average over the noise in the outer shell. After this transformation the resultant equations are shown diagrammatically 
a)

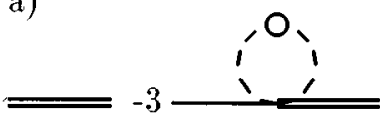
$-4 \frac{-0-1}{=-1}=$
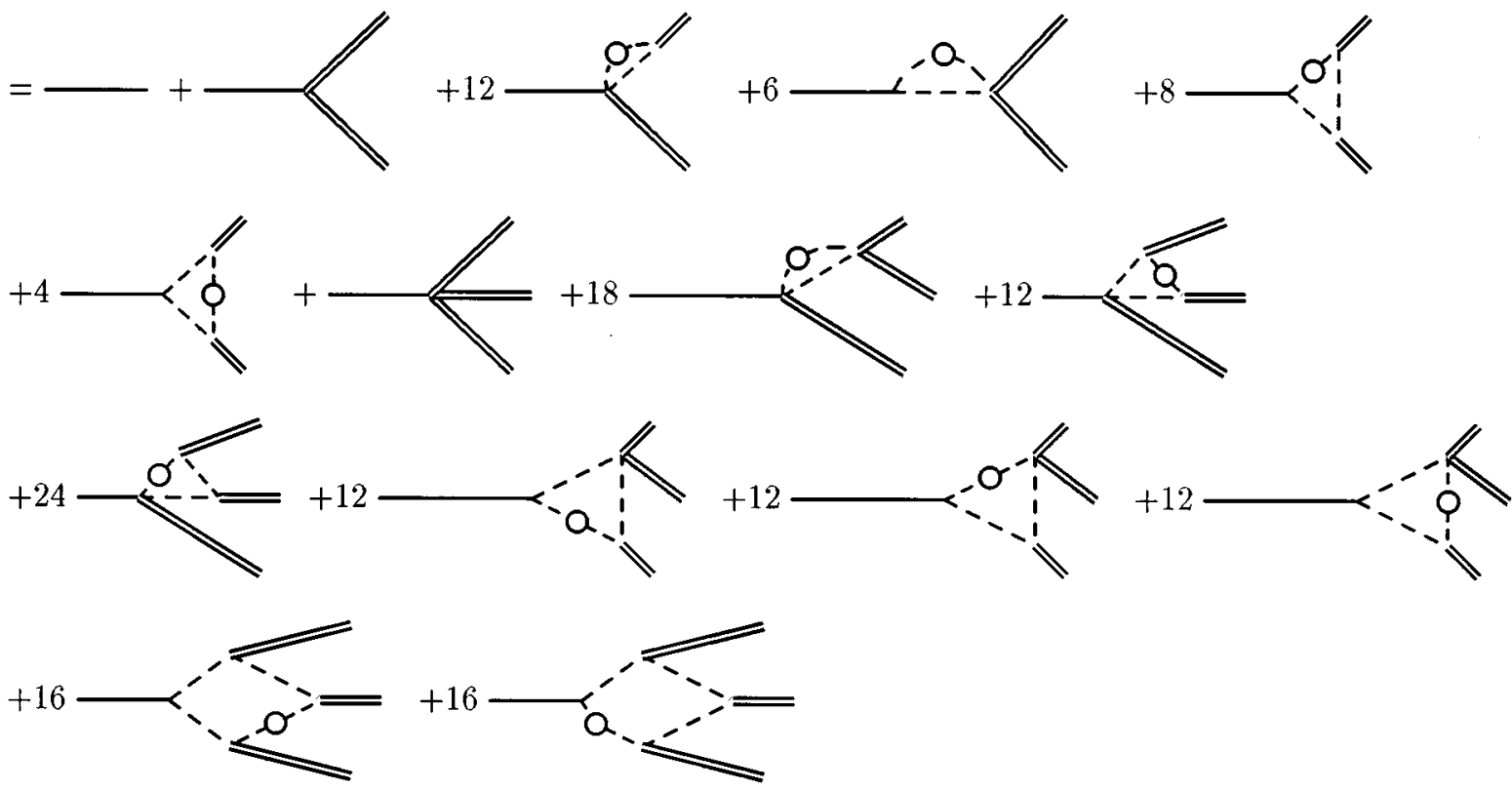

b)

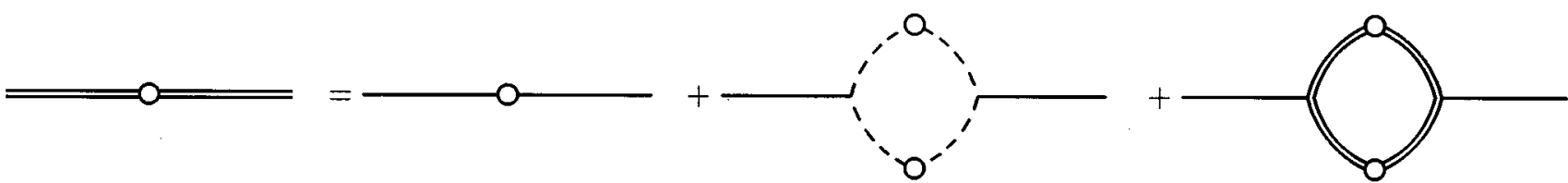

FIG. 2. Diagrams obtained after the first step of the DRG transformation. Now continuous lines correspond to the inner shell, whereas dashed lines correspond to the outer shell. A comparison with Fig. 1 allows one to define new coefficients. Observe that the new averages affect only the outer shell. The notation has been simplified with respect to Fig. 1, suppressing the symbol $\times$ at the end of the vertices and also the arrows.

in Fig. 2. It is clear that we can obtain new equations that are formally equivalent to the initial ones, Eqs. (21) and (25), defining the new coefficients as the original ones plus the corresponding integrals over the outer shell. With the noise correlation (26) these integrals can be easily computed in the hydrodynamic limit $(\vec{k} \rightarrow 0, \omega \rightarrow 0)$, as it is shown in the Appendix, and then the coefficients transform according to

$$
\begin{gathered}
\Gamma \rightarrow \Gamma, \\
D \rightarrow D\left[1+3 \frac{I_{d} \Gamma \lambda_{3}}{D^{3}}-4 \frac{I_{d} \Gamma \lambda_{2}^{2}}{D^{4}}\right], \\
\lambda_{2} \rightarrow \lambda_{2}\left[1-18 \frac{I_{d} \Gamma \lambda_{3}}{D^{3}}+12 \frac{I_{d} \Gamma \lambda_{2}^{2}}{D^{4}}\right],
\end{gathered}
$$

$$
\lambda_{3} \rightarrow \lambda_{3}\left[1-18 \frac{I_{d} \Gamma \lambda_{3}}{D^{3}}+72 \frac{I_{d} \Gamma \lambda_{2}^{2}}{D^{4}}-32 \frac{I_{d} \Gamma \lambda_{2}^{4}}{D^{5} \lambda_{3}}\right]
$$

where

$$
I_{d}(l)=\frac{2 S_{d}}{(2 \pi)^{d}} \frac{1-e^{-l(d-4)}}{d-4} \Lambda^{d-4}
$$

and $S_{d}$ is the complete solid angle in $d$ dimensions. However, the new equations are only defined in the inner shell $0<k<e^{-l} \Lambda$. The second step allows us to recover the original Brillouin zone $0<k<\Lambda$ by rescaling the equations using transformation (16), which in Fourier space writes

$$
\overrightarrow{k^{\prime}}=e^{l} \vec{k}, \quad \omega^{\prime}=e^{z l} \omega, \quad E^{\prime}=e^{-(\chi+z+d) l} E .
$$


The combined effect of both transformations, in the limit $l \rightarrow 0$, constitutes an infinitesimal DRG transformation, which gives the flow equations of the parameters in parameter space. In these flow equations instead of $\lambda_{2}$ and $\lambda_{3}$ it is suitable to use the dimensionless coupling constants $\bar{\lambda}_{2}$ and $\bar{\lambda}_{3}$, given by

$$
\bar{\lambda}_{2}^{2}=\frac{I_{d}^{(1)} \Gamma \lambda_{2}^{2}}{D^{4}}, \quad \bar{\lambda}_{3}=\frac{I_{d}^{(1)} \Gamma \lambda_{3}}{D^{3}},
$$

where $I_{d}^{(1)}=\left(d I_{d} / d l\right)_{l=0}=\left[2 S_{d} /(2 \pi)^{d}\right] \Lambda^{d-4}$. Then

$$
\begin{gathered}
\frac{d \Gamma}{d l}=\Gamma[2 z-2 \chi-d], \\
\frac{d D}{d l}=D\left[z-2-4 \bar{\lambda}_{2}^{2}+3 \bar{\lambda}_{3}\right], \\
\frac{d \bar{\lambda}_{2}}{d l}=\bar{\lambda}_{2}\left[\frac{4-d}{2}+20 \bar{\lambda}_{2}^{2}-24 \bar{\lambda}_{3}\right], \\
\frac{d \bar{\lambda}_{3}}{d l}=\bar{\lambda}_{3}\left[4-d+84 \bar{\lambda}_{2}^{2}-27 \bar{\lambda}_{3}-32 \frac{\bar{\lambda}_{2}^{4}}{\bar{\lambda}_{3}}\right] .
\end{gathered}
$$

We are interested in the invariance of the parameters under DRG transformations. This means that we have to look for the fixed points of the flow equations; if we write Eqs. (31) as $d \alpha_{i} / d l=g_{i}\left(\cdots \alpha_{j} \cdots\right)$, where $\alpha_{j}$ represents any coefficient, then the fixed points verify $g_{i}\left(\cdots \alpha_{j}^{*} \cdots\right)=0 \quad \forall i$. Considering $D \neq 0$ and $\Gamma \neq 0$, we obtain four algebraic equations with four unknowns $\chi, z, \bar{\lambda}_{2}$, and $\bar{\lambda}_{3}$; their solutions will give us the fixed points of the transformation, $\bar{\lambda}_{2}^{*}$ and $\bar{\lambda}_{3}^{*}$, as well as the values of the exponents $z$ and $\chi$ that guarantee that the DRG transformation leads to a scale-free behavior. Notice that the particular values of $\Gamma$ and $D$ play no role in the existence and location of the fixed points. We can also find the stability of the fixed points under small perturbations using a linear stability analysis: the fixed point $\left\{\alpha_{j}^{*}\right\}$ is stable (i.e., an attractor) if all the eigenvalues (or their real parts) of the matrix $\partial g_{i} / \partial \alpha_{j}$ evaluated at this fixed point are negative.

The results are the following: for $d>4$ one obtains six different fixed points, but the only stable one corresponds to

$$
\chi=\frac{\epsilon}{2}, \quad z=2, \quad \bar{\lambda}_{2}^{*}=\bar{\lambda}_{3}^{*}=0,
$$

where as usual $\epsilon$ is defined as $\epsilon=d_{c}-d=4-d$. This is the trivial or Gaussian fixed point, which gives a normal (or Brownian) diffusive behavior because of the vanishing of the coupling constants. The values of the exponents do not correspond with those of the Edwards-Wilkinson model, used in the study of surface growth, because the noise correlation is different [34]. For $d<4$ this fixed point becomes unstable and the only stable one is

$$
\chi=\frac{7}{18} \epsilon, \quad z=2-\frac{\epsilon}{9}, \quad \bar{\lambda}_{2}^{*}=0, \quad \bar{\lambda}_{3}^{*}=\frac{\epsilon}{27},
$$

which was unstable for $d>4$. In this case the diffusion is anomalous; to be more precise, the fact that $z<2$ gives a superdiffusive behavior in the hydrodynamic limit. Note that the one-loop expansion in the intensity of the noise $\Gamma$ gives a nontrivial fixed point that is expressed as a perturbation of the Gaussian one in a first-order $\epsilon$ expansion. Observe also that the breaking of symmetry does not modify the value of the fixed point obtained without taking into account the even coupling constant $\lambda_{2}$ [24]. Moreover, the fact that the nontrivial fixed point is an attractor of the dynamics contrasts with equilibrium critical phenomena, where this point is stable only along one direction. In this fact lies the difference between fine tuning of parameters for equilibrium systems at the critical point and self-organization towards criticality for nonequilibrium processes.

Now we know the attractors in the parameter space, but this is not enough in our case; since our stochastic equation (13) is derived directly from the discrete rules of the BTW and Zhang models, we also need to know the basins of attraction of the stable fixed points and whether our initial conditions, that is, the initial values of the coefficients corresponding to our physical problem, are inside these basins. These values for the dimensionless coupling constants (30) can be calculated from Eqs. (14) and (15) and they are

$$
\left(\bar{\lambda}_{2}^{0}\right)^{2}=\frac{1}{4} \frac{I_{d}^{(1)} \Gamma^{0}}{\alpha^{2} E_{c}^{2}} \frac{f^{(2)}(K)^{2}}{f^{(1)}(K)^{4}}, \quad \bar{\lambda}_{3}^{0}=\frac{1}{6} \frac{I_{d}^{(1)} \Gamma^{0}}{\alpha^{2} E_{c}^{2}} \frac{f^{(3)}(K)}{f^{(1)}(K)^{3}},
$$

result that also holds for $K=0$, where we obtain $\bar{\lambda}_{2}^{0}=0$ even for the Zhang model. The superscript 0 indicates the initial value of the coefficient, that is, its value before any renormalization. As we have no restriction for $\Gamma^{0}$ (except that it has to be small) and $K$ can take any arbitrary real value, this implies that the initial dimensionless coupling constants will be defined in the region

$$
\bar{\lambda}_{3}^{0}<\frac{2}{3}\left(\bar{\lambda}_{2}^{0}\right)^{2},
$$

having used for $f(x)$ the explicit form given by Eq. (9).

Clearly, the stable fixed point for $d<4$ [Eq. (33)] is outside the region of initial conditions defined by Eq. (35). It will be of maximum interest, however, to know whether or not these conditions will drive the system towards the nontrivial fixed point. We first consider $K=0$, which implies $\bar{\lambda}_{2}^{0}=0$, corresponding to the case studied in Ref. [24]. For $d<4$ one gets a different behavior depending on the sign of $\bar{\lambda}_{3}^{0}$. Figure 3 shows that when $\bar{\lambda}_{3}^{0}$ is positive it flows towards the stable fixed point $\bar{\lambda}_{3}^{*}=\epsilon / 27$, giving a dynamical exponent $z=2-\epsilon / 9$. A negative $\bar{\lambda}_{3}^{0}$, which is our case of physical interest, flows away. An exact solution of Eq. (31d) with $\bar{\lambda}_{2}=0$ gives that $\bar{\lambda}_{\underline{3}}$ would reach $-\infty$ in a finite $l$ and then would reappear as $\bar{\lambda}_{3}=\infty$, then being under the attraction of the nontrivial fixed point. However, our one-loop calculation forces the flow of the coupling constant along the parameter space to stay of order $\epsilon$, and one cannot sustain the validity of the preceding description. Then it is not possible to predict the renormalization of $\bar{\lambda}_{3}$. It will be either renormalized to $\bar{\lambda}_{3}^{*}$ or other fixed points will appear along the flow (corre- 
$d<4$

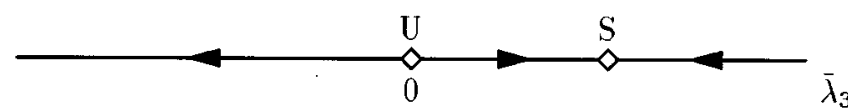

$d>4$

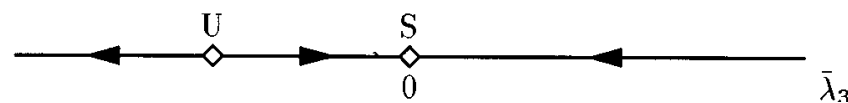

FIG. 3. Flow in $\bar{\lambda}_{3}$ space when only this nonlinear term is taken into account. The squares correspond to the stable $(S)$ and unstable $(U)$ fixed points and the arrows show the flow under DRG transformations.

sponding to strong coupling and not given by the one-loop $\epsilon$ expansion). Therefore, the fixed point given by Eq. (33), although it is an attractor, is unreachable from our initial conditions $\left(\bar{\lambda}_{3}^{0}<0\right)$. For that reason the conclusions of Ref. [24] were incomplete. On the other hand, above the upper critical dimension the system evolves towards the trivial fixed point $\bar{\lambda}_{3}^{*}=0$ giving a diffusive behavior with $z=2$ provided that $\bar{\lambda}_{3}^{0}$ is not too negative (see Fig. 3). This behavior of the fixed point $\bar{\lambda}_{3}^{*}$ as a function of $\epsilon$ corresponds to a transcritical bifurcation.

Now, by introducing the alternative regularization ( $K$ $\neq 0$ ), we will see the effect of the symmetry breaking. First of all, we insist that the stable fixed points are the same as for $K=0$, due to the fact that $\bar{\lambda}_{2}$ renormalizes to zero. Moreover, as can be seen in Fig. 4, where we have plotted the flow lines of Eq. (31) obtained by numerical integration, the basin of attraction of the nontrivial fixed point is delimited by the parabola

$$
\bar{\lambda}_{3}=\frac{4}{7} \bar{\lambda}_{2}^{2}
$$

which is also a particular solution of the flow equations, regardless of the value of $d$. This parabola is inside the region defined by Eq. (35), and this fact implies that the new regularization makes it possible to reach the attractor for $d<4$ starting in the region of physical meaning. Using Eqs. (34) and (36) together with Eq. (9), one gets that the condition to converge towards the nontrivial fixed point is $K^{2}>\frac{7}{2}$. Then, the parameter that breaks the symmetry in the regularization of the step function, which, in principle, was arbitrary, determines the behavior of the system in the hydrodynamic limit.

For $d>4$ the flow is more complex because of the six fixed points, but the result is that convergence towards the Gaussian one also happens for our initial conditions, as Fig. 5 shows. The linear stability analysis of the fixed points gives the same results as the numerical integration shown in the figure. However, this linear analysis fails for $d=4$, where all the fixed points collapse towards the Gaussian one. It is by means of the numerical integration that we verify that it is an attractor for the region above the parabola given by Eq. (35), but for the region below it is a repeller. This strange behavior appears because in $d=4$ we are at the bifurcation point.

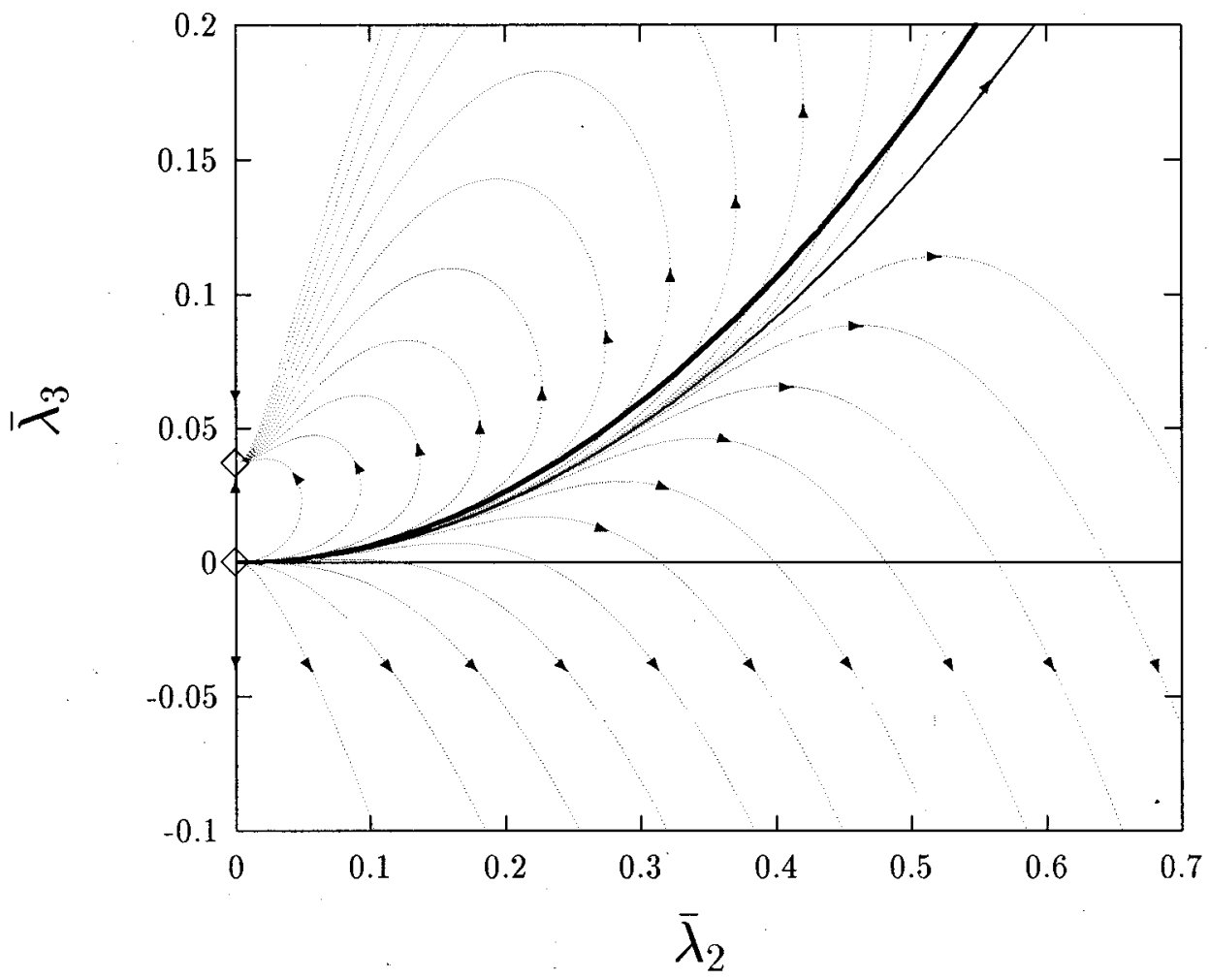

FIG. 4. Flow in $\left(\bar{\lambda}_{2}, \bar{\lambda}_{3}\right)$ space for $d=3$ when both nonlinear terms are taken into account. In general, for any $d<4$ the results are qualitatively the same. Dots correspond to the numerical integration of Eqs. (31c) and (31d) and the thin line is Eq. (36), which clearly delimits the basin of attraction of the nontrivial fixed point, as it is seen in the plot. Below the continuous thick line the values of the parameters correspond to our physical situation Eq. (35). Squares correspond to the fixed points. Observe that for $\bar{\lambda}_{2}=0$ we obtain the same results as in Fig. 3. 


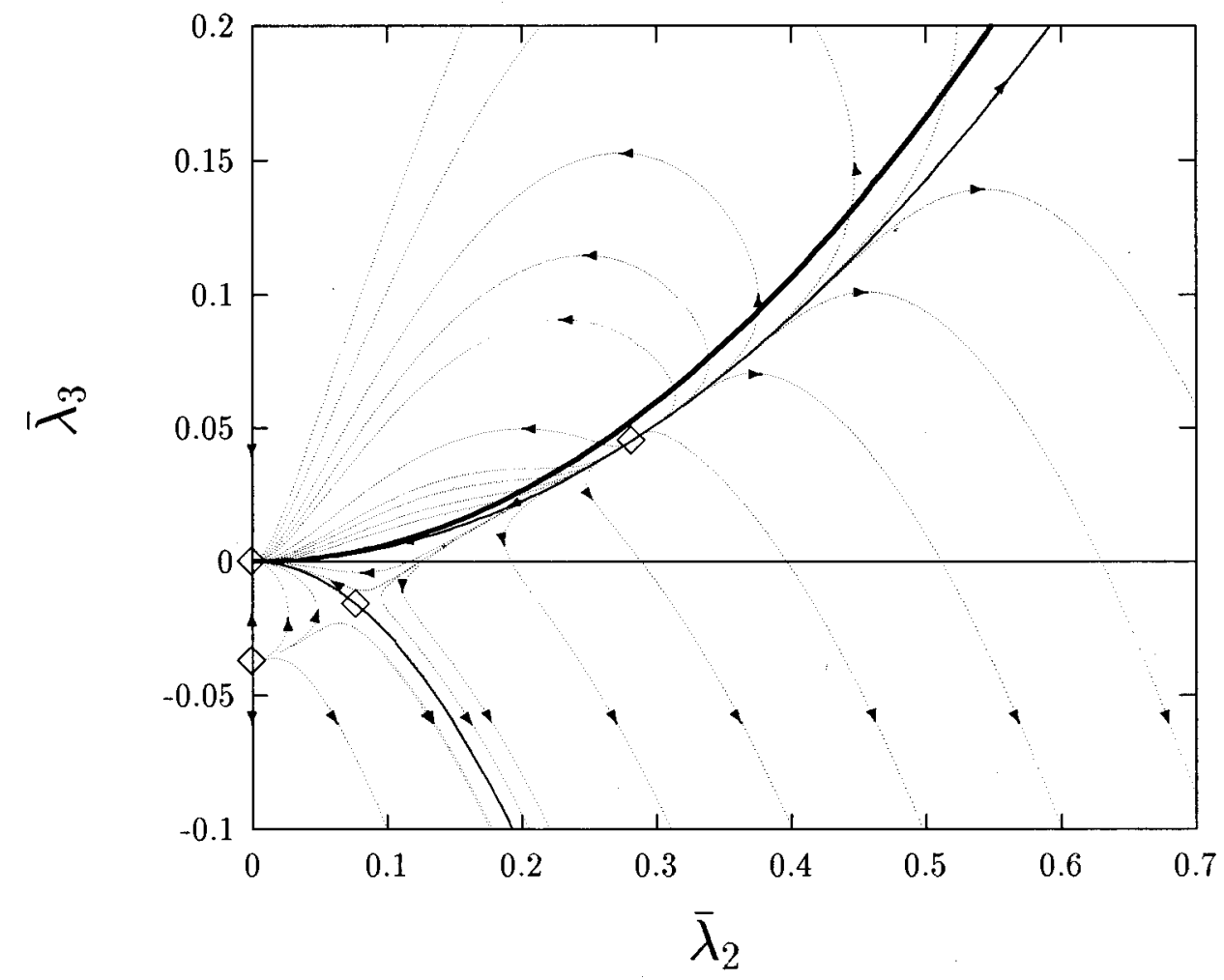

FIG. 5. Same as Fig. 4, but for $d=5$. The results hold for $d>4$. Only four of the six fixed points are shown because of the symmetry of the flow lines. In this case, the curve $\bar{\lambda}_{3}=-\frac{8}{3} \bar{\lambda}_{2}^{2}$, represented by another thin line, is the repulsive branch of the saddle point.

In Ref. [24] it was shown for the BTW model and $K=0$ that $\lambda_{2 n}=0$, whereas for the Zhang model, although the even coupling constants do not vanish, it was argued that their flow equations became decoupled from the odd ones in the limit $\beta \rightarrow \infty$. This fact enabled us to establish the same universality class for both models and to deal with only odd terms in Eq. (13). Then, an expansion in the number of coupling constants was performed, whose extrapolation compares well with the results of the simulations [2,5]. Note that in the simulations one computes the dynamical exponent relating the characteristic length and lifetime of the avalanches, whereas within the DRG framework one computes the dynamical exponent from the fluctuations of the order parameter [35]. The agreement between these calculations confirms the basic scaling hypothesis that in both cases length and time are related by means of the same exponent. However, the problem of this calculation was that the nontrivial fixed point was unreachable for the original equation.

In our approach, due to the symmetry breaking, we have to consider also the effect of even coupling constants. In the present work we have dealt with a restricted problem with only the lower-order even and odd coupling constants $\lambda_{2}$ and $\lambda_{3}$, showing that $\lambda_{2}$ renormalizes to zero, supporting the calculation of Ref. [24]. Then the stable fixed points are not modified by the presence of an even coupling constant in the model, but due to the symmetry breaking that we have introduced, the nontrivial one is an attractor in the parameter space when the parameters corresponding to the real model are taken into account. This behavior should be the same for any even coupling constant; actually, preliminary calculations including $\lambda_{4}$ and $\lambda_{5}$ in Eq. (19) make us suspect that all even coupling constants renormalize to zero. This fact means that in the hydrodynamic limit the solution of both models has to be symmetric under parity transformations of the order parameter; then, for the BTW model the DRG restores the broken symmetry, whereas for the Zhang model we conclude that its asymmetric nature is irrelevant in the behavior at large distances and long times. Therefore, this validates the extrapolation performed in [24] since now we have show that the symmetry breaking makes the stable fixed points reachable, when starting in the region of physical interest in the space of parameters. Let us finally mention that in a recent work, Ghaffari and Jensen [36] performed a different extrapolation of the same results, which show better agreement with large-scale simulations and with real-space renormalization calculations for the dynamical exponent [23]. It is noticeable than the same technique has been applied to the study of the effect of dissipation in a uniformly driven BTW model [37].

\section{CONCLUSION}

We have studied analytically two models that show selforganized criticality. The difference between them is that the second one (BTW) is symmetric under a parity transformation, whereas the first (Zhang) model is not. From the microscopic rules one writes a effective long-wavelength equation involving the threshold condition, which enters into the equation through a step function, making the equation unapproachable under this form. We have introduced a regularization of the step function that breaks the symmetry of the BTW model. After a power-series expansion, the equation is suitable for the application of the dynamic renormalization group, although it contains an infinite number of relevant coupling constants. As a consequence, one has to truncate at some point the expansion in the coupling constants. The results only make sense if it is possible to extrapolate the values of the exponents up to an infinite number of coupling 
constants. We obtain the fixed points of the transformation in parameter space and study carefully their stability and basins of attraction. Then we find that with this regularization it is possible to reach the nontrivial fixed point for $d<4$, which was unreachable in a previous work, where symmetry was not broken. This means that in the hydrodynamic limit the models display scale invariance. Moreover, in this limit we obtain a symmetric behavior under parity transformations for both models and therefore the recovery of the broken symmetry for the BTW model and the irrelevance of this symmetry for Zhang's model. Although we have dealt with a simplified version of the problem, we expect this behavior to be the same for the complete problem in the sense that all even coupling constants renormalize to zero, validating the calculation of Ref. [24]. The application of this technique should also be useful for other kinds of problems in which one deals with thresholds or with an infinite number of nonlinear terms, for instance, interface dynamics. Moreover, the DRG calculation performed is interesting because it provides an example showing how important it is to know not only the stable fixed points of a DRG transformation but also their basins of attraction. It is remarkable that a simple symmetry breaking can solve the problem of the inaccessibility of the attractors in parameter space.

The fact that the parameter that breaks the symmetry determines the behavior in the hydrodynamic limit is difficult to understand and we believe that it is an artificiality introduced in the calculation by the truncation in the couplingconstant expansion. We expect that higher orders in this expansion will give a behavior independent of the $K$ value.

\section{ACKNOWLEDGMENTS}

The authors wish to acknowledge A. Arenas, R. Cuerno, and C. J. Pérez for very fruitful discussions. A.C. thanks the Spanish Ministerio de Educación y Cultura for financial support. This work has been supported by CICyT of the Spanish Government, Grants Nos. PB92-0863 and PB94-0897.

\section{APPENDIX}

Here we present further details about the derivation of Eqs. (27), which give the transformation of the parameters after the first step of the DRG. Our starting points are Eqs. (21) and (25), i.e., the equations for $E(\vec{k}, \omega)$ and $\left\langle E(\vec{k}, \omega) E\left(\vec{k}^{\prime}, \omega^{\prime}\right)\right\rangle$. As we have already mentioned, these equations are only defined for $0<k<\Lambda$. The DRG procedure consists in splitting the momentum space into two shells: an inner one, with $0<k<\Lambda e^{-l}$, and an outer one, with $\Lambda e^{-l}<k<\Lambda$. Then the magnitudes that depend on $\vec{k}$, like the energy $E$, split as

$$
\begin{aligned}
E(\vec{k}, \omega) & =E^{<}(\vec{k}, \omega)+E^{>}(\vec{k}, \omega) \\
& =E(\vec{k}, \omega) \Theta\left(\Lambda e^{-l}-k\right)+E(\vec{k}, \omega) \Theta\left(k-\Lambda e^{-l}\right),
\end{aligned}
$$

where $\Theta(x)$ is again the Heaviside step function. This equality defines $E^{<}(\vec{k}, \omega)$ as the corresponding part of the energy in the inner shell, whereas $E^{>}(\vec{k}, \omega)$ is the same, but defined in the outer shell. This separation also holds for the bare propagator $G_{0}$ and the noise $\eta$.
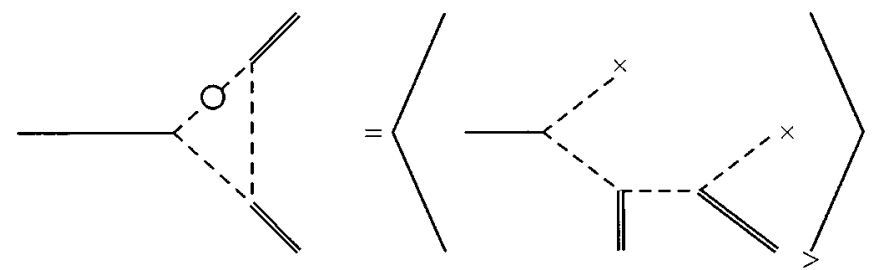

FIG. 6. Diagram computed in the Appendix as an example. The angular brackets stand for an average over the outer shell.

The DRG procedure eliminates the modes of the outer shell, within the same philosophy as the Kadanoff transformation in real space. Then one is only interested in $E^{<}(\vec{k}, \omega)$ and $\left\langle E^{<}(\vec{k}, \omega) E^{<}\left(\vec{k}^{\prime}, \omega^{\prime}\right)\right\rangle$, whose equations turn out to be equivalent to Eqs. (21) and (25), but with additional terms due to the coupling between the two shells, via the convolution products. The fact that $E^{>}(\vec{k}, \omega)$ appears in the inner-shell equations allows a perturbative expansion in the form $E^{>}(\vec{k}, \omega)=G_{0}^{>}(\vec{k}, \omega) \eta^{>}(\vec{k}, \omega)+\cdots$ [using the equivalent of Eq. (21), but in the outer shell]. Then the noise in the outer shell enters into the equation for $E^{<}(\vec{k}, \omega)$. A similar perturbative expansion is done for $\left\langle E^{<}(\vec{k}, \omega) E^{<}\left(\vec{k}^{\prime}, \omega^{\prime}\right)\right\rangle$. By averaging over $\eta^{>}(\vec{k}, \omega)$, the contribution of the fast modes is eliminated from the inner shell. This is done up to one-loop order in the perturbative expansion, that is, the lowest order in the intensity of the noise $\Gamma$, which implies that it has to be small enough. This tedious calculation becomes more appealing using the diagrams of Fig. 1 instead of the corresponding equations. After this process, the relevant diagrams that survive the averaging are shown in Fig. 2.

As an example let us consider one of them, shown in Fig. 6 and denoted by $V(\vec{k}, \omega)$ :

$$
\begin{aligned}
V(\vec{k}, \omega)= & \left\langle\frac{-\lambda_{2} k^{2}}{(2 \pi)^{d+1}} G_{0}^{<}(\vec{k}, \omega) \int d^{d} q d \Omega G_{0}^{>}(\vec{k}-\vec{q}, \omega-\Omega)\right. \\
& \times G_{0}^{>}(\vec{q}, \Omega) \eta^{>}(\vec{q}, \Omega) \frac{-\lambda_{2}(\vec{k}-\vec{q})^{2}}{(2 \pi)^{d+1}} \\
& \times \int d^{d} q^{\prime} d \Omega^{\prime} G_{0}^{>}\left(\vec{k}-\vec{q}-\overrightarrow{q^{\prime}}, \omega-\Omega-\Omega^{\prime}\right) \\
& \times E^{<}\left(\overrightarrow{q^{\prime}}, \Omega^{\prime}\right) \frac{-\lambda_{2}\left(\vec{k}-\vec{q}-\overrightarrow{q^{\prime}}\right)^{2}}{(2 \pi)^{d+1}} \\
& \times \int d^{d} q^{\prime \prime} d \Omega^{\prime \prime} G_{0}^{>}\left(\vec{k}-\vec{q}-\overrightarrow{q^{\prime}}-\overrightarrow{q^{\prime \prime}}, \omega-\Omega-\Omega^{\prime}\right. \\
& \left.-\Omega^{\prime \prime}\right) \eta^{>}\left(\vec{k}-\vec{q}-\overrightarrow{q^{\prime}}-\overrightarrow{q^{\prime \prime}}, \omega-\Omega-\Omega^{\prime}-\Omega^{\prime \prime}\right) \\
& \left.\times E^{<}\left(\overrightarrow{q^{\prime \prime}}, \Omega^{\prime \prime}\right)\right\rangle_{>},
\end{aligned}
$$

where the symbol \langle\rangle$_{>}$stands for an average over the outer shell. Using the noise correlation given by Eq. (26), we can integrate over $\Omega, \Omega^{\prime \prime}$, and $\overrightarrow{q^{\prime \prime}}$, and then we have 


$$
\begin{aligned}
V(\vec{k}, \omega)= & -\frac{2 \lambda_{2}^{3} \Gamma}{(2 \pi)^{2 d+1}} k^{2} G_{0}^{<}(\vec{k}, \omega) \int d^{d} q^{\prime} d \Omega^{\prime} \\
& \times E^{<}\left(\overrightarrow{q^{\prime}}, \Omega^{\prime}\right) E^{<}\left(\vec{k}-\vec{q}^{\prime}, \omega-\Omega^{\prime}\right) \int d^{d} q \\
& \times G_{0}^{>}(\vec{k}-\vec{q}, \omega) G_{0}^{>}(\vec{q}, 0)(\vec{k}-\vec{q})^{2} \\
& \times G_{0}^{>}\left(\vec{k}-\vec{q}-\vec{q}^{\prime}, \omega-\Omega^{\prime}\right)\left(\vec{k}-\vec{q}-\vec{q}^{\prime}\right)^{2} G_{0}^{>}(\vec{q}, 0) .
\end{aligned}
$$

As the bare propagator is a known function, given by Eq. (23), we are also able to perform the integral over $\vec{q}$, that is,

$$
\begin{aligned}
\int d^{d} q[]= & \int d^{d} q G_{0}^{>}(\vec{k}-\vec{q}, \omega) G_{0}^{>2}(\vec{q}, 0)(\vec{k}-\vec{q})^{2} \\
& \times G_{0}^{>}\left(\vec{k}-\vec{q}-\overrightarrow{q^{\prime}}, \omega-\Omega^{\prime}\right)\left(\vec{k}-\vec{q}-\overrightarrow{q^{\prime}}\right)^{2}
\end{aligned}
$$

This integral is a function of $\vec{k}, \omega, \overrightarrow{q^{\prime}}$, and $\Omega^{\prime}$. However, we are going to evaluate it in the hydrodynamic limit by taking $\vec{k}, \overrightarrow{q^{\prime}} \rightarrow 0$ and $\omega, \Omega^{\prime} \rightarrow 0$. Then

$$
\begin{aligned}
\int d^{d} q[] & =\int d^{d} q q^{4} G_{0}^{>2}(-\vec{q}, 0) G_{0}^{>2}(\vec{q}, 0) \\
& =\frac{S_{d}}{D^{4}} \int_{\Lambda e^{-l}}^{\Lambda} q^{d-5} d q=\frac{S_{d}}{D^{4}} \frac{\Lambda^{d-4}}{d-4}\left(1-e^{-l(d-4)}\right) .
\end{aligned}
$$

It is easy to check that this result is also valid for $d=4$. We have used the explicit form of the bare propagator (23) and also that $d^{d} q=S_{d} q^{d-1} d q$, with $S_{d}$ the complete solid angle in $d$ dimensions, that is, the area of a unit $(d)$ sphere. Then, by making use of Eq. (28), we obtain

$$
\int d^{d} q[]=\frac{(2 \pi)^{d} I_{d}(l)}{2 D^{4}}
$$

and substituting into Eq. (A3),

$$
\begin{aligned}
V(\vec{k} \rightarrow 0, \omega \rightarrow 0) \rightarrow & -\frac{\lambda_{2} k^{2}}{(2 \pi)^{d+1}} G_{0}^{<}(\vec{k}, \omega) \\
& \times\left(E^{<} * E^{<}\right)(\vec{k}, \omega) I_{d}(l) \frac{\lambda_{2}^{2} \Gamma}{D^{4}}
\end{aligned}
$$

It is clear from Fig. 2 that after the first step of the DRG we have the same diagrams as at the beginning (Fig. 1), but defined only in the inner shell, plus many diagrams of the same type as the one in Fig. 6. These diagrams, which contain integrals over the outer shell, renormalize the other diagrams that are only defined in the inner shell. For instance, if we consider the diagram

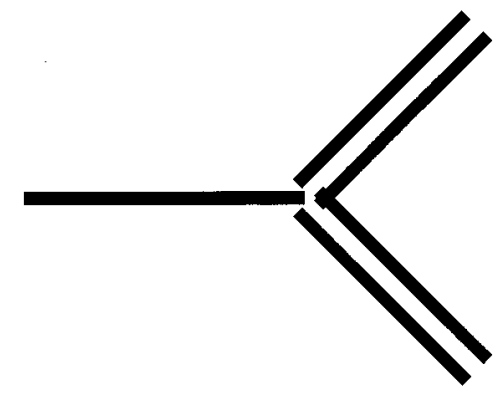

$$
=-\frac{\lambda_{2} k^{2}}{(2 \pi)^{d+1}} G_{0}^{<}(\vec{k}, \omega)\left(E^{<*} E^{<}\right)(\vec{k}, \omega)
$$

and compare it with Eq. (A7), we observe only an additional term $I_{d}(l) \lambda_{2}^{2} \Gamma / D^{4}$ that comes from the outer-shell integration. So the diagram shown in Fig. 6 contributes to the renormalization of Eq. (A8), that is, it renormalizes the coupling constant $\lambda_{2}$. As Fig. 2(a) shows, the diagram in Fig. 6 appears eight times in the perturbative expansion and the new $\lambda_{2}$, after the first step of the transformation, will be modified by

$$
\lambda_{2} \rightarrow \lambda_{2}\left(1+8 I_{d}(l) \frac{\lambda_{2}^{2} \Gamma}{D^{4}}+\cdots\right)
$$

In the same way one can perform the outer-shell integrals of the rest of diagrams in Fig. 2(a). A general result for its contribution to the renormalization of any coupling constant $\lambda_{n}$ or to the diffusion coefficient $D$ (which will be referred to here also as $-\lambda_{1}$ ) is given by

$$
(-1)^{v-1} I_{d}(l) \frac{\Gamma}{D^{v+1}} \frac{\prod_{m=1}^{v} \lambda_{b(m)}}{\lambda_{B}},
$$

where $v$ is the number of vertices each diagram has, [3 for our example (since the dashed line in Fig. 6 forms a triangle) ], $b(m)$ is the number of branches of the $m$ th vertex (2 for each one in the example), and $B$ is the number of branches of the diagram that is renormalized ( 2 in the example) and fulfills $B=\sum_{m=1}^{v} b(m)-v-1$. Note that the magnitude in Eq. (A10) is dimensionless. Using this equation and Fig. 2(a) the derivation of Eqs. (27b) $-(27 d)$ is then straightforward.

For the renormalization of the intensity of the noise $\Gamma$ we have only one diagram, the dashed one in Fig. 2(b). It is immediate to see that the integral over the outer shell (the value does not matter) is multiplied by a factor $k^{2} k^{\prime 2}$. Then

$$
\Gamma \rightarrow \Gamma\left(1+A k^{2} k^{\prime 2} \frac{\lambda_{2}^{2} \Gamma}{D^{4}}+\ldots\right),
$$

where $A$ is simply a numeric factor, and hence, in the hydrodynamic limit and up to one-loop order, the intensity of the noise is not renormalized after the first step of the DRG, as Eq. (27a) states. 
[1] P. Bak, C. Tang, and K. Wiesenfeld, Phys. Rev. Lett. 59, 381 (1987); Phys. Rev. A 38, 364 (1988).

[2] Y.-C. Zhang, Phys. Rev. Lett. 63, 470 (1989).

[3] L. Pietronero, P. Tartaglia, and Y.-C. Zhang, Physica A 173, 22 (1991).

[4] Z. Fodor and I. M. Janosi, Phys. Rev. A 44, 1386 (1991).

[5] A. Díaz-Guilera, Phys. Rev. A 45, 8551 (1992).

[6] L. P. Kadanoff, S. R. Nagel, L. Wu, and S. Zhou, Phys. Rev. A 39, 6524 (1989).

[7] S. S. Manna, Physica A 179, 249 (1991).

[8] I. M. Janosi, Phys. Rev. A 42, 769 (1990).

[9] S. S. Manna, L. B. Kiss, and J. Kertész, J. Stat. Phys. 61, 923 (1990).

[10] H. J. S. Feder and J. Feder, Phys. Rev. Lett. 66, 2669 (1991).

[11] Z. Olami, H. J. S. Feder, and K. Christensen, Phys. Rev. Lett. 68, 1244 (1992).

[12] K. Christensen, Z. Olami, and P. Bak, Phys. Rev. Lett. 68, 2417 (1992).

[13] Z. Olami and K. Christensen, Phys. Rev. A 46, R1720 (1992).

[14] A. Corral, C. J. Pérez, A. Díaz-Guilera, and A. Arenas, Phys. Rev. Lett. 74, 118 (1995).

[15] C. J. Pérez, A. Corral, A. Díaz-Guilera, K. Christensen, and A. Arenas, Int. J. Mod. Phys. B 10, 1111 (1996).

[16] K. Christensen, A. Corral, V. Frette, J. Feder, and T. Jøssang, Phys. Rev. Lett. 77, 107 (1996).

[17] M. Paczuski and S. Boettcher, Phys. Rev. Lett. 77, 111 (1996).

[18] T. Hwa and M. Kardar, Phys. Rev. Lett. 62, 1813 (1989).

[19] G. Grinstein, D.-H. Lee, and S. Sachdev, Phys. Rev. Lett. 64, 1927 (1990).
[20] G. Grinstein, in Scale Invariance, Interfaces, and NonEquilibrium Dynamics, edited by A. McKane et al. (Plenum, New York, 1995).

[21] J. M. Carlson, J. T. Chayes, E. R. Grannan, and G. H. Swindle, Phys. Rev. Lett. 65, 2547 (1990).

[22] P. Bantay and I. M. Janosi, Phys. Rev. Lett. 68, 2058 (1992).

[23] L. Pietronero, A. Vespignani, and S. Zapperi, Phys. Rev. Lett. 72, 1690 (1994); A. Vespignani, S. Zapperi, and L. Pietronero, Phys. Rev. E 51, 1711 (1995).

[24] A. Díaz-Guilera, Europhys. Lett. 26, 177 (1994).

[25] D. Forster, D. R. Nelson, and M. J. Stephen, Phys. Rev. A 16, 732 (1977).

[26] E. Medina, T. Hwa, M. Kardar, and Y.-C. Zhang, Phys. Rev. A 39, 3053 (1989).

[27] A.-L. Barabási and H. E. Stanley, Fractals Concepts in Surface Growth (Cambridge University Press, Cambridge, 1995).

[28] S. Maslov (private communication).

[29] A. Corral and A. Díaz-Guilera (unpublished).

[30] K. Christensen (private communication).

[31] A. Díaz-Guilera, Fractals 1, 963 (1993).

[32] M. Kardar, G. Parisi, and Y.-C. Zhang, Phys. Rev. Lett. 56, 889 (1986).

[33] For a way of calculating a scaling function see T. Hwa and E. Frey, Phys. Rev. A 44, R7873 (1991).

[34] S. F. Edwards and D. R. Wilkinson, Proc. R. Soc. London Ser. A 381, 17 (1982).

[35] A. Giacometti and A. Díaz-Guilera (unpublished).

[36] P. Ghaffari and H. Jensen (unpublished).

[37] P. Ghaffari, S. Lise, and H. Jensen (unpublished). 